Environmental and Ecological Statistics manuscript No.

(will be inserted by the editor)

\title{
A Test of Independence Between Nonhomogeneous Poisson Processes and its Application to Heat Wave Modeling
}

\author{
Jesús Abaurrea · Jesús Asín · Ana C. Cebrián \\ Received: date / Accepted: date
}

\begin{abstract}
The main contribution of this work is a test to check the independence between nonhomogeneous Poisson processes. It is developed for the three-process case, but it can be generalized to any number of processes. Moreover, it can be applied not only to Poisson processes but to any point process which can be simulated. It is a Monte Carlo test if the parameters describing the processes are known, and a parametric bootstrap test in the most usual case of unknown parameters. A simulation study shows that the size of the test is close to the nominal one. The power is also analyzed, considering two approaches for generating dependent nonhomogeneous processes and different levels of dependence, with satisfactory results in all the cases.

This test is a valuable tool in the validation analysis of a common Poisson shock process. For the bivariate case, this process can be decomposed into three indepen-

J. Abaurrea · J. Asín · A.C. Cebrián

Dpto. Métodos Estadísticos. Universidad de Zaragoza

Tel.: +34 976762885

E-mail: acebrian@unizar.es
\end{abstract}


2

dent Poisson processes, and the independence of the three marginal processes has to be checked for validating the model. As an application, the modeling of the occurrence process of extreme heat events in daily maximum and minimum temperatures is described.

Keywords Parametric bootstrap - Independence test - Point process - Bivariate Poisson process · Extreme event

\section{Introduction}

This work aims to develop a test to check the independence between nonhomogeneous point processes, in particular, nonhomogeneous Poisson processes $(\mathrm{NH}-$ PPs). As far as we know, there does not exist a test of independence between onedimensional NHPPs. Tests of independence between point processes have been studied in the framework of marked spatial point processes, see Schlather et al. (2004), Eckel et al. (2007), Guan and Afshartous (2007) and Comas et al. (2010). There also exist quite a few functions aiming to quantify the dependence between spatial point processes, such as the ones suggested by Diggle (1983) and van Lieshout and Baddeley (1999), and some of them are also used in time point processes (Doss 1989). Some of the previous independence tests can be adapted to time point processes, but little work has been done with nonhomogeneous (NH) processes. Cuzick and Edwards (1990) suggested a test to detect clustering, and Allard et al. (2001) a test of local independence based on approximate isotropy for $\mathrm{NH}$ spatial point processes. Baddeley et al. (2000) and Marcon and Puech (2009) developed a generalization of the Ripley's $K$ function to an inhomogeneous spatial setting. 
The need of developing this test stemmed from a real problem, the modelling of extreme heat events. A bivariate model is needed in order to model the distribution of the occurrence of extremes in maximum and minimum daily temperatures, accounting for the dependence between the two signals. A reasonable model is a bivariate nonhomogenoeus common Poisson shock process, which can be described by three independent NHPPs. The proposed test is necessary to check the independence between the three marginal processes.

The interest of characterizing extreme heat events in a bivariate framework is clear. First, because heat waves are known to produce serious impacts on regional economies, ecosystems and human health. Secondly, because Palecki et al. (2001), Grintzevitch (2006) and other studies about the effect of extreme heat on mortality rates found that both maximum and minimum temperatures have an impact on human health. As a consequence, an increasing number of heat wave definitions include information on both temperatures, see for example Plummer et al. (1999), or the definition of Excessive Heat Watch by the U.S. National Weather Service.

Section 2 describes the proposed test to check independence. In Section 3, a simulation study of the size and the power of this test is carried out. Section 4 shows an application, the modeling process of the occurrence of the extreme heat events in Zaragoza (Spain). The most relevant conclusions are summarized in Section 5. 


\section{Methodology}

2.1 A test to check the independence of several nonhomogeneous Poisson processes

One might think that a way to simplify the problem of testing the independence of $\mathrm{NH}$ processes is to transform them into homogeneous ones as a previous step. However, it is noteworthy that with conditional intensities $\lambda^{*}(t)$, this is not a feasible approach since the dependence structure disappears under simultaneous random time transformations of the type $t^{\prime}=\int_{0}^{t} \lambda^{*}(u) d u$ (Daley and Vere-Jones 2003, prop. 7.4.VI).

Hence, we propose a test to check the independence between one-dimensional $\mathrm{NH}$ point processes, conditionally on their corresponding intensities. Let us denote $\left(N_{x}, N_{y}, N_{z}\right)$ a vector of three point processes with intensities $\lambda_{x}(t), \lambda_{y}(t)$ and $\lambda_{z}(t)$, where $n_{x}, n_{y}$ and $n_{z}$ points have been observed. If dependence exists, it is reasonable to assume that it will manifest itself in events occurring close to each other, so we tried to generalize the crossed nearest neighbor distance ideas to the $\mathrm{NH}$ case. These distances only consider the dependence between the occurrence of points, but dependence can also manifest in the non occurrence. To account for this aspect, each point is linked to the time interval until its previous neighbor, which will be called the time interval of the point. We define the close point relation $\rightsquigarrow$ between two points in two different processes as follows: a point in a process is close to a point in another process, if the time interval of the first overlaps the time interval of the second. For example, in the left plot in Figure 1, $t x_{i} \rightsquigarrow t y_{j}$ and in the right one, $t y_{j} \rightsquigarrow t z_{k}$ and $t y_{j} \rightsquigarrow t z_{k+1}$. 
Then, given the vector process $\left(N_{x}, N_{y}, N_{z}\right)$, the set of close points of $t x_{i}$ in $N_{x}$, the big point in Figure 1 (left), is defined as,

$$
S_{x_{i}}=\left\{\left(t y_{j}, t z_{k}\right) \in N_{y} \times N_{z}: t x_{i} \rightsquigarrow t y_{j}, t y_{j} \rightsquigarrow t z_{k}\right\}
$$

In the example in Figure $1, S_{x_{i}}=\left\{\left(t y_{j}, t z_{k}\right),\left(t y_{j}, t z_{k+1}\right)\right\}$.

To check if there is a longer dependence in the process, the close point relation could be broadened by including the previous and/or the following points to the overlapping intervals: $t y_{j-1}$ and $t y_{j+1}$ in Figure 1 (left) and $t z_{k-1}$ and $t z_{k+2}$ in Figure 1 (right). If no relevant difference is observed, it will be concluded that the actual dependence has been properly taken into account. Otherwise, it should be checked if more points must be added to capture the dependence structure.

If only the points with overlapping time intervals are considered, the close point relation is symmetric, but if more points are added, it is not. For example, in Figure 1 (left) $t x_{i} \rightsquigarrow t y_{j-1}$ but $t y_{j-1}$ is not close to $t x_{i}$. In that case, and if there is not an intrinsic order between the processes, all the permutations resulting from interchanging the order of the processes should be considered to build the sets of close points.

The underlying idea of the test is to compare, for each point of $N_{x}$, the behavior of its set of close points in the observed vector process $\left(N_{x}, N_{y}, N_{z}\right)$, and in simulated processes with the same characteristics (same $N_{x}$ process and second and third processes with the same intensities) and mutually independent components. If the observed behavior is significantly different, the independence will be rejected. The multivariate character of the points and the nonhomogeneity make it difficult to obtain a statistic with a known probability distribution. However, the distribution can be 
6

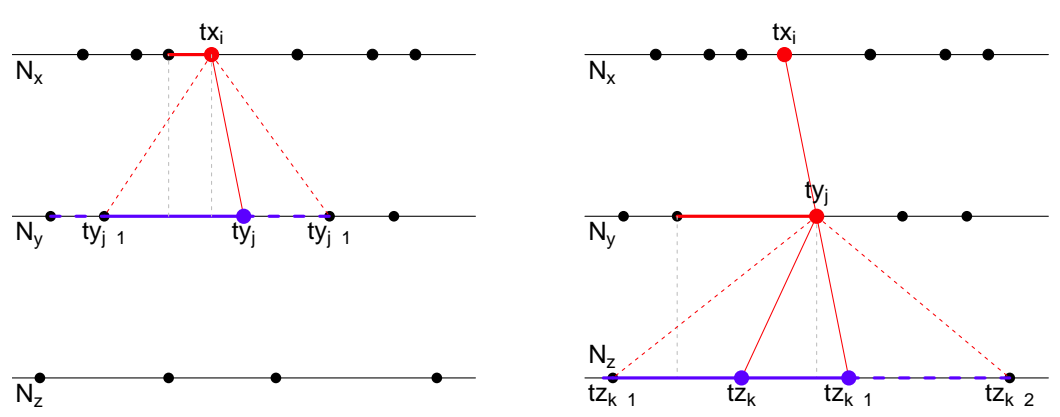

Fig. 1 Definition of the set of close points of a point $t x_{i}$. The dashed lines to $t y_{j-1}$ and $t y_{j+1}$ in the left plot and to $t z_{k-1}$ and $t z_{k+2}$ in the right one, mark the previous and the following points of the overlapping intervals.

obtained using a Monte Carlo approach if the intensities $\lambda_{y}(t)$ and $\lambda_{z}(t)$ are known, or a parametric bootstrap if they have to be estimated.

The steps of the test are the following:

Step 1. For each point $t x_{i}$ in $N_{x}$, its set of close points in the observed vector process $\left(N_{x}, N_{y}, N_{z}\right), S_{x_{i}}$, is built and $\bar{d} x_{i}$, the mean of the distances $\left|t y_{j}-t x_{i}\right|+$ $\left|t z_{k}-t x_{i}\right|$ of the pairs in that set, is calculated as a summary.

Step 2.1. A vector process $\left(N_{x}, N_{y}^{*}, N_{z}^{*}\right)$ is generated, where $N_{y}^{*}$ and $N_{z}^{*}$ are independent processes with intensities $\lambda_{y}(t)$ and $\lambda_{z}(t)$, also independent of the observed process $N_{x}$.

Step 2.2. For each point $t x_{i}$ in $N_{x}$, its set of close points in the simulated vector process $\left(N_{x}, N_{y}^{*}, N_{z}^{*}\right), S_{x_{i}}^{*}$, is built and $\bar{d} x_{i}^{*}$, the mean of the distances $\left|t y_{j}^{*}-t x_{i}\right|+$ $\left|t z_{k}^{*}-t x_{i}\right|$, calculated.

Step 2 is repeated $n_{S}$ times so that, for each $t x_{i}$, a set of one observed and $n_{S}$ simulated distances is obtained. Under the independence hypothesis, this should be 
a sample of i.i.d. observations and $p x_{i}$, the sample percentile of $\bar{d} x_{i}$, an observation from a uniform distribution.

The final result of this process is a series of $n_{x}$ (the number of points in $N_{x}$ ) percentiles $p x_{i}$. These percentiles should be $U(0,1)$ under the null hypothesis, but it is not guaranteed that they are uncorrelated. The serial correlation comes from the possible presence of some equal pairs $\left(t y_{j}, t z_{k}\right)$ in the sets of close points of nearby points $t x_{i}$ and $t x_{l}$.

Kolmogorov-Smirnov (KS) is a well-tried statistic for testing whether a sample stems from a given distribution, for example the uniform one. Although the distribution of this statistic in a correlated sample is not known, its empirical distribution can be obtained from the previously simulated trajectories. To that aim, the uniform KS statistic for the percentile sample obtained from each simulated vector of independent processes ( $D_{n_{x}}$ herein) is calculated, so that a size $n_{S}$ sample of $D_{n_{x}}$ under the null hypothesis is obtained. The p-value of the test is calculated from that sample. As in the standard KS test, the null hypothesis is rejected if $d>d_{1-\alpha}$, where $d$ is the observed value of $D_{n_{x}}$ and $d_{1-\alpha}$ is the $1-\alpha$ percentile of the statistic distribution under the null hypothesis. The simulated NHPP trajectories are used to calculate both the percentiles and the distribution of $D_{n_{x}}$, so that the computing time cost is not noticeably increased.

2.2 Validity of the test

When the parameters ( $\lambda_{y}$ and $\lambda_{z}$ in this case) are known, this type of tests are Monte Carlo tests and they are exact (Dufour 2006). However, in most real problems the in- 
tensities are unknown. In that case, the p-value is obtained by a parametric bootstrap, where the unknown parameters $\lambda_{y}$ and $\lambda_{z}$ of the bootstrap data generating process (DGP) are substituted by their estimators.

Most of the results for parametric bootstrap tests are developed for pivotal or asymptotically pivotal statistics, whose distribution does not depend on the unknown parameters, see Beran (1988), Davidson and MacKinnon (1999) and Davidson and MacKinnon (2006b). In our test, the structure of the statistic makes it difficult to prove if it is pivotal, or to make it pivotal by a scale transformation. On the other hand, the nuisance parameters $\lambda_{y}$ and $\lambda_{z}$ do not appear in the null hypothesis $H_{0}$ : processes are independent, unlike most parametric bootstrap tests (Beran 1988) (Andrews 1997). That makes the study of the test properties simpler.

First, in order to be valid, the bootstrap distribution of the statistic must mimic its real distribution under the null, even though the data were generated under the alternative hypothesis (Andrews 1997). If $\lambda_{y}$ and $\lambda_{z}$ are estimated using the likelihood function of the marginal processes, with no restrictions, they are estimated under the null, and the previous condition is guaranteed.

Concerning the bootstrap distribution, Davidson and MacKinnon (2006a) remark that 'it is desirable to get as good an estimate as possible of the true DGP for the bootstrap DGP'. This requires a good estimation method which provides consistent estimators, such as maximum likelihood, and a thorough validation analysis of the models used to estimate the parameters. Given $n_{x}$, the distribution $G_{n_{x}}\left(d, F\left(\hat{\lambda}_{y}, \hat{\lambda}_{z}\right)\right)$ of $D_{n_{x}}$, considered as a functional of the distribution of the DGP $F\left(\hat{\lambda}_{y}, \hat{\lambda}_{z}\right)$, is contin- 
uous. Then, according to Horowitz (2001), if MLE $\hat{\lambda}_{y}$ and $\hat{\lambda}_{z}$ are used, it is expected that $G_{n_{x}}\left(d, F\left(\hat{\lambda}_{y}, \hat{\lambda}_{z}\right)\right)$ will be close to $G_{n_{x}}\left(d, F\left(\lambda_{y}, \lambda_{z}\right)\right)$.

To show empirically the closeness of $G_{n_{x}}\left(d, F\left(\hat{\lambda}_{y}, \hat{\lambda}_{z}\right)\right)$ and $G_{n_{x}}\left(d, F\left(\lambda_{y}, \lambda_{z}\right)\right)$ distributions, the following simulation study is done. We generate three independent NHPP $N_{x}, N_{y}$ and $N_{z}$ with given intensities $\lambda_{x}(t), \lambda_{y}(t)$ and $\lambda_{z}(t)$, which play the role of observed processes. First, applying the test with these intensities, a sample of size $n_{S}$ of $D_{n_{x}}$ is obtained and the distribution $G_{n_{x}}\left(d, F\left(\lambda_{y}, \lambda_{z}\right)\right)$ calculated from that sample. Then, using $N_{y}$ and $N_{z}$ and adequate covariates, the estimators $\hat{\lambda}_{y}$ and $\hat{\lambda}_{z}$ are calculated, and applying the test with them, the distribution $G_{n_{x}}\left(d, F\left(\hat{\lambda}_{y}, \hat{\lambda}_{z}\right)\right)$ is obtained. A KS test is used to check if the distribution of both samples is the same. Since the simulation study aims to check the behavior of the bootstrap test assuming that good estimators are used, we only consider the cases where the fitted model satisfies some basic validation analysis and the real intensities are covered by the $95 \%$ confidence intervals estimated from the model. The validation analysis consists of checking the distribution and the uncorrelation of the uniform residuals of the NHPP, see section 4.5 for more details.

The simulation study is performed with the intensities,

$$
\begin{aligned}
& \lambda_{x}(t)=\exp \left[-56-48 \cos (2 \pi t)-23 \sin (2 \pi t)-0.02 t+0.001 t^{2}\right] \\
& \lambda_{y}(t)=\exp \left[-8-2 \cos (2 \pi t)+3 \sin (2 \pi t)+0.03 t+0.0002 t^{2}\right] \\
& \lambda_{z}(t)=\exp \left[-10.5-6 \cos (2 \pi t)-3 \sin (2 \pi t)+0.01 t+0.0003 t^{2}\right]
\end{aligned}
$$

which include the most usual features of a real evolution in time: a seasonal term (one harmonic) and a polynomial trend (quadratic in this case). The qqplot comparing the 
Table 1 Summary of the p-values from the KS test comparing the empirical distributions $G_{n_{x}}\left(d, F\left(\hat{\lambda}_{y}, \hat{\lambda}_{z}\right)\right)$ and $G_{n_{x}}\left(d, F\left(\lambda_{y}, \lambda_{z}\right)\right)$

\begin{tabular}{lccccc}
\hline KS p-value $>$ & 0.05 & 0.25 & 0.5 & 0.75 & 0.95 \\
Proportion & 0.950 & 0.714 & 0.504 & 0.302 & 0.056 \\
\hline
\end{tabular}

samples from the distributions $G_{n_{x}}\left(d, F\left(\hat{\lambda}_{y}, \hat{\lambda}_{z}\right)\right)$ and $G_{n_{x}}\left(d, F\left(\lambda_{y}, \lambda_{z}\right)\right)$ is shown in Figure 2 and the corresponding KS p-value is 0.26 . This analysis is repeated 500 times. The resulting p-values of the KS test comparing both distributions are summarized in Table 1. In most cases high values are obtained, and the equality of the distributions is rejected, at a $\alpha=0.05$ significance level, in approximately $5 \%$ of the cases. Hence, there is no evidence that the distribution of the test statistic changes when the real parameters are substituted by their ML estimators.

The study about $G_{\infty}$, the asymptotic distribution of the statistic is not relevant in this test. In each case, the bootstrap distribution of the statistic is calculated for the given sample size $n_{x}$ with a low time computing cost and, consequently, the approximation provided by $G_{\infty}$ is not needed. As far as we know, there does not exist any exact or asymptotic test to check the independence of NHPPs, and comparative analyses of the performance and the order of approximation of the proposed test cannot be carried out.

\section{Size and power of the test}

This section summarizes the main results from an analysis of the size and the power of the test. All the experiments are based on 1000 trials, and in each trial the p-value is calculated with $n_{S}=1000$. Given the equality of the distributions $G_{n_{x}}\left(d, F\left(\hat{\lambda}_{y}, \hat{\lambda}_{z}\right)\right)$ 


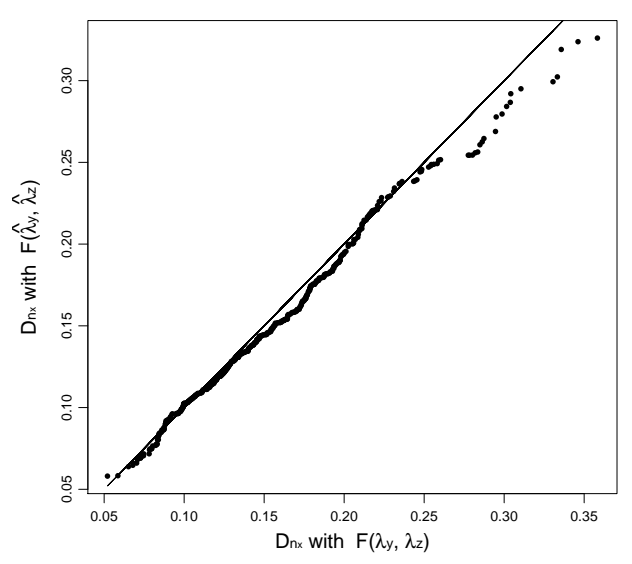

Fig. 2 QQplot comparing the distributions $G_{n_{x}}\left(d, F\left(\hat{\lambda}_{y}, \hat{\lambda}_{z}\right)\right)$ and $G_{n_{x}}\left(d, F\left(\lambda_{y}, \lambda_{z}\right)\right)$

and $G_{n_{x}}\left(d, F\left(\lambda_{y}, \lambda_{z}\right)\right)$ and in order to simplify the analysis, the properties of the test are studied in its Monte Carlo version.

\subsection{Size of the test}

Table 2 summarizes, for nominal levels $\alpha=0.05$ and 0.10 , the rate of rejection under the null hypothesis, i.e. given three independent processes with different intensities and observation period $T$. In the first case, the intensities are the fitted ones in the application described in section $4, \hat{\lambda}_{(1)}(t), \hat{\lambda}_{(2)}(t)$ and $\hat{\lambda}_{(12)}(t)$ and $T=8415$. The second and third cases are HPPs with intensities equal to the mean of these fitted intensities and $T=8415$ and $T=4000$, respectively. In the last case, three NHPPs with a linearly increasing intensity function $f(t)$ and $T=8000$ are considered. The estimated sizes are close to the nominal levels for both the homogeneous and the $\mathrm{NH}$ 
12

processes. The size of the test does not seem to be influenced neither by the length $T$, nor by the shape of the intensity function.

\subsection{Power of the test}

The alternative hypothesis (three dependent processes) is very general, and it is not possible to systematically study it. Two approaches are considered here, the first one generates dependent Poisson processes using two queues in tandem. The second generates dependent point processes, not Poisson, using marks based on a Markov chain. In both cases, the power of the test is studied according to the degree of the induced dependence.

Queue approach. Burke's theorem states that, in a stable stationary system, the output process from any $M / M / m$ queue is a PP with the same rate $\lambda$ as the input process (Kleinrock 1975). In a tandem of $M / M / 1$ queues with exponential time service of rates $\mu_{1}$ and $\mu_{2}$, the input and the output processes of the first queue and the output of the second queue are three dependent PPs. This approach, considering a constant input intensity $\lambda=0.01$, is used to generate dependent HPPs.

Table 2 Size of the test for different DGPs and nominal levels.

\begin{tabular}{llllll}
\hline$T$ & $\lambda_{x}(t)$ & $\lambda_{y}(t)$ & $\lambda_{z}(t)$ & $\alpha=0.10$ & $\alpha=0.05$ \\
\hline 8415 & $\hat{\lambda}_{(12)}(t)$ & $\hat{\lambda}_{(2)}(t)$ & $\hat{\lambda}_{(1)}(t)$ & 0.101 & 0.046 \\
8415 & $\overline{\hat{\lambda}}_{(12)}$ & $\overline{\hat{\lambda}}_{(2)}$ & $\overline{\hat{\lambda}}_{(1)}$ & 0.095 & 0.052 \\
4000 & $\overline{\hat{\lambda}}_{(12)}$ & $\overline{\hat{\lambda}}_{(2)}$ & $\overline{\hat{\lambda}}_{(1)}$ & 0.100 & 0.052 \\
8000 & $f(t)$ & $f(t)$ & $f(t)$ & 0.115 & 0.049 \\
\hline
\end{tabular}


There is a relationship between the utilization factors of the two queues, $\rho_{1}$ and $\rho_{2}\left(\rho_{i}=\lambda / \mu_{i}\right)$, and the degree of dependence between the input/output processes. This relation is not simple since utilization factors near zero lead to high correlations, whereas a high variability in the correlation is observed with higher factors (lower than 1).

To study the power according to the degree of dependence, the empirical Pearson correlation coefficient between the inter-arrival times of each pair of processes is categorized into four levels, very low (L), low (l), high (h) and very high (H), depending on which interval the coefficients lie in: $[0,0.25),[0.25,0.5),[0.50,0.75)$ and $[0.75$, 1]. The different combinations of correlation levels between the first and the second process and between the second and the third are considered. The power of the test at levels $\alpha=0.10$ and $\alpha=0.05$, using processes with $n_{x}=100$ points, are shown in Table 3. The critical value of the Pearson correlation coefficient at a significance level $\alpha=0.01$ for a sample of size 100 is 0.25 , so that correlations in level $\mathrm{L}$ can be considered null. The generated trajectories which have not the desired correlation values are discarded. The values of $\mu_{1}$ and $\mu_{2}$ are selected in order to get discarding rates as low as possible, column Disc. in the table.

The power for $\alpha=0.10$ is higher than 0.70 when very high or high correlations are observed. The power decreases when two of the three processes are independent or almost independent (levels Hl, HL and hl). However, the test performance perceptibly improves when the sample size increases to $n_{x}=200$, see the right columns of the table. Even in those cases where the estimated power is not very high, the p-value 
Table 3 Power of the test for HPPs with different correlation levels, $M / M / 1$ queue approach

\begin{tabular}{|c|c|c|c|c|c|c|c|c|c|}
\hline \multirow[b]{2}{*}{ Level } & \multirow[b]{2}{*}{$\rho_{1}$} & \multirow[b]{2}{*}{$\rho_{2}$} & \multirow[b]{2}{*}{ Disc. } & \multicolumn{3}{|c|}{$n_{x}=100$} & \multicolumn{3}{|c|}{$n_{x}=200$} \\
\hline & & & & $\alpha=0.10$ & $\alpha=0.05$ & $p v_{0.5}$ & $\alpha=0.10$ & $\alpha=0.05$ & $p v_{0.5}$ \\
\hline $\mathrm{HH}$ & 0.4 & 0.4 & $18 \%$ & 0.990 & 0.981 & 0.000 & - & - & - \\
\hline $\mathrm{Hh}$ & 0.4 & 0.6 & $40 \%$ & 0.900 & 0.824 & 0.007 & - & - & - \\
\hline $\mathrm{Hl}$ & 0.4 & 0.8 & $57 \%$ & 0.600 & 0.442 & 0.070 & 0.895 & 0.812 & 0.007 \\
\hline HL & 0.4 & 0.9 & $58 \%$ & 0.531 & 0.381 & 0.089 & 0.836 & 0.720 & 0.014 \\
\hline hh & 0.6 & 0.6 & $54 \%$ & 0.701 & 0.544 & 0.042 & 0.951 & 0.898 & 0.003 \\
\hline hl & 0.6 & 0.8 & $64 \%$ & 0.359 & 0.234 & 0.180 & 0.622 & 0.491 & 0.053 \\
\hline $\mathrm{hL}$ & 0.6 & 0.9 & $77 \%$ & 0.220 & 0.122 & 0.306 & 0.427 & 0.306 & 0.143 \\
\hline 11 & 0.8 & 0.8 & $76 \%$ & 0.163 & 0.082 & 0.460 & 0.292 & 0.182 & 0.236 \\
\hline $1 \mathrm{~L}$ & 0.8 & 0.9 & $70 \%$ & 0.114 & 0.061 & 0.450 & 0.172 & 0.094 & 0.366 \\
\hline
\end{tabular}

distribution is clearly concentrated on small values, as the median of the p-values $p v_{0.5}$ shows.

Generation of NHPPs with the queue approach. The queue approach can also be used to generate dependent NHPPs applying the result by Keilson and Servi (1994): the output process from a $M(t) / G / 1$ queue is also a PP with a $\mathrm{NH}$ intensity equal to the convolution $\lambda_{\text {out }}(t)=\lambda_{\text {inp }}(t) * f_{\mu}(t)$, where $\lambda_{\text {inp }}(t)$ is the input intensity and $f_{\mu}(x)$ the density function of the service time, in this case an exponential distribution of rate $\mu$.

In the $\mathrm{NH}$ case the correlation between the processes cannot be easily characterized, and the power cannot be studied according to the correlation level. It can be expected that, as in the homogeneous case, high utilization factors will often generate 
processes which are not under the alternative hypothesis. By this reason, the power analysis for $\mathrm{NH}$ intensities is restricted to the case $\rho_{1}=\rho_{2}=0.4$ where, in the homogeneous case, $99 \%$ of the trajectories had a high or very high correlation. We defer to the Markov chain approach, a more thorough study of the power according to the dependence in the $\mathrm{NH}$ case.

In this experiment, the test performance is analyzed in relation to the shape of the intensity, including the homogeneous case as a reference. In all the cases the mean intensity is 0.011 and $T=8415$, so that the number of points in each trajectory is $n_{x} \approx 100$. The first $\mathrm{NH}$ input intensity is the sum of the fitted rates in the data application, $\lambda_{o}(t)=\hat{\lambda}_{(1)}(t)+\hat{\lambda}_{(2)}(t)+\hat{\lambda}_{(12)}(t)$, which shows a complex evolution, see Figure 4. $\lambda_{o_{R}}(t)$ and $\lambda_{o_{A}}(t)$ are two transformations of $\lambda_{o}(t)$, which reduce and amplify, respectively, its variability:

$$
\begin{aligned}
& \lambda_{o_{R}}(t)=\left(\lambda_{o}(t)-\lambda_{c}\right) / 2+\lambda_{c} \\
& \lambda_{o_{A}}(t)=0.01 \lambda_{o}(t)\left(\lambda_{o}(t)<\lambda_{c}\right)+1.14 \lambda_{o}(t)\left(\lambda_{o}(t)>\lambda_{c}\right)
\end{aligned}
$$

being $\lambda_{c}=\overline{\lambda_{o}(t)}=0.011$, and 0.01 and 1.14 the most extreme factors that keep positive the intensity function. A process with input intensity $\lambda_{p s}$, defined by a linearly increasing function of time, and the homogeneous intensity $\lambda_{c}$ are also considered. In all the cases $\bar{\rho}_{1}=\bar{\rho}_{2}=0.4$ and their minimum and maximum utilization values are shown in Table 4.

The homogeneous case shows the highest rejection rates but, even for the most complex intensities, the results are satisfactory. The power improves with the sample size, as the results for a period of length $2 T$ with intensity $\lambda_{o, 2 T}(t)$, obtained by concatenating twice $\lambda_{o}(t)$, show. 
Table 4 Rejection rates of the test for NHPPs, $M(t) / M / 1$ queue approach, T=8415

\begin{tabular}{llllllll}
\hline Int. & $\max \left(\rho_{1}\right)$ & $\max \left(\rho_{2}\right)$ & $\min \left(\rho_{1}\right)$ & $\min \left(\rho_{2}\right)$ & $\alpha=0.10$ & $\alpha=0.05$ & $p v_{0.5}$ \\
\hline$\lambda_{c}$ & 0.4 & 0.4 & 0.4 & 0.4 & 0.974 & 0.950 & 0.001 \\
$\lambda_{o}(t)$ & 19.3 & 4.3 & $6 \mathrm{e}-6$ & $9 \mathrm{e}-3$ & 0.881 & 0.791 & 0.009 \\
$\lambda_{o_{A}}(t)$ & 22.1 & 4.9 & $6 \mathrm{e}-8$ & $7 \mathrm{e}-4$ & 0.823 & 0.703 & 0.018 \\
$\lambda_{o_{R}}(t)$ & 9.9 & 2.4 & 0.2 & 0.2 & 0.957 & 0.918 & 0.002 \\
$\lambda_{p s}(t)$ & 0.8 & 0.8 & $1 \mathrm{e}-4$ & $1 \mathrm{e}-2$ & 0.848 & 0.740 & 0.013 \\
$\lambda_{o, 2 T}(t)$ & 19.3 & 4.3 & $6 \mathrm{e}-6$ & $9 \mathrm{e}-3$ & 0.996 & 0.987 & 0.000 \\
\hline
\end{tabular}

Markov chain approach. In this study, dependent homogeneous and NH point processes are generated by marking a PP using a three-state Markov chain. The processes defined by the marks are not Poisson, since the generated marks are dependent observations (Isham 1980). However, as previously mentioned, the test can be applied to any point process which can be simulated. A transition matrix $P=\left(p_{i j}\right)$ with three equal rows leads to three independent point processes, and the more similar the rows of $\mathrm{P}$, the less dependent the resulting processes. Two different dependence measures are considered in the power analysis. The first one is the spectral gap $S G$, which assesses the convergence speed of $P$ to a matrix $P_{I}$ with all the rows equal to $\left(\pi_{1}, \pi_{2}, \pi_{3}\right)$, the stationary distribution of $P . S G$ takes values in $[0,1]$.

The results for four doubly stochastic Markovian matrices (M1 to M4) with an increasing convergence speed are shown in the upper rows of Table 5. M1 is a matrix which does not converge, $S G=0$, and defines a completely deterministic marking processes. The aforementioned intensity $\lambda_{o}(t)$ and $T=8415\left(n_{x} \approx 100\right)$ are used to generate the underlying PP. The study is also run using the intensities $\lambda_{c}$ and $\lambda_{o, 2 T}(t)$ 
(with period length $2 T$ ). The power at significance levels $\alpha=0.05$ and $\alpha=0.10$ is summarized in the table, and the effect of $S G$ on the performance of the test is clear. The power is satisfactory for $S G<0.6$. For matrices closer to the independence, such as M4, the results are poorer but they improve when the sample size increases. Slightly better results are observed in the homogeneous case.

For matrices with the same convergence speed, we consider a second dependence measure related to the time until leaving the current state $i$, which has a Geometric(1$p_{i i}$ ) distribution. We define $D_{I}=\sum_{i=1}^{3} p_{i i} / 3$, which varies from 0 to 1 , being $1 / 3$ the value for the limit matrix $P_{I}$. If the test behaves properly, the further $D_{I}$ is from $1 / 3$, the better the performance of the test should be. The lower part of Table 5 shows the doubly stochastic matrices $M 3 b$ and $M 3 c$ obtained by permuting the probabilities in $M 3$, which have the same $S G$ as $M 3$ but different $D_{I}$. $D_{I}$ values far from 1/3 lead to a better power and, since $1 / 3$ is not centered on the $D_{I}$ sample space, the increase is not symmetric around that value. The results for the other three permuted matrices of M3 and for permuted matrices of M2 and M4, not shown here, confirm that the test performance for matrices with the same $S G$ and $D_{I}$ is equivalent.

\section{Data application}

In order to jointly model the distribution of the occurrence of extreme heat events in maximum and minimum temperatures, taking into account the dependence between the two signals, a bivariate model is needed. The seasonality and a possible time evolution of the phenomenon require a nonstationary process. We propose a nonhomogeneous common Poisson shock process (NHCPSP) which, for the bivariate case, 
Table 5 Power analysis: dependent processes generated by the Markov chain approach, $T=8415$

\begin{tabular}{|c|c|c|c|c|c|c|c|}
\hline \multicolumn{2}{|c|}{ Markov matrix } & SG & $D_{I}$ & $\alpha$ & $\lambda_{o}(t)$ & $\lambda_{c}$ & $\lambda_{o, 2 T}(t)$ \\
\hline$M 1=$ & $\left(\begin{array}{lll}0 & 1 & 0 \\
0 & 0 & 1 \\
1 & 0 & 0\end{array}\right)$ & 0.00 & & $\begin{array}{l}0.05 \\
0.10\end{array}$ & $\begin{array}{l}1 \\
1\end{array}$ & $\begin{array}{l}1 \\
1\end{array}$ & - \\
\hline$M 2=$ & $\left(\begin{array}{lll}0.7 & 0.2 & 0.1 \\
0.1 & 0.7 & 0.2 \\
0.2 & 0.1 & 0.7\end{array}\right)$ & 0.44 & & $\begin{array}{l}0.05 \\
0.10\end{array}$ & $\begin{array}{l}0.987 \\
0.994\end{array}$ & $\begin{array}{c}0.997 \\
1\end{array}$ & - \\
\hline$M 3=$ & $\left.\begin{array}{cccc}0.6 & 0.3 & 0.1 \\
0.1 & 0.6 & 0.3 \\
0.3 & 0.1 & 0.6\end{array}\right)$ & 0.56 & 0.6 & $\begin{array}{l}0.05 \\
0.10\end{array}$ & $\begin{array}{l}0.751 \\
0.828\end{array}$ & $\begin{array}{l}0.857 \\
0.919\end{array}$ & $\begin{array}{l}0.937 \\
0.969\end{array}$ \\
\hline$M 4=$ & $\left(\begin{array}{ccc}0.5 & 0.2 & 0.3 \\
0.3 & 0.5 & 0.2 \\
0.2 & 0.3 & 0.5\end{array}\right)$ & 0.74 & & $\begin{array}{l}0.05 \\
0.10\end{array}$ & $\begin{array}{l}0.418 \\
0.526\end{array}$ & $\begin{array}{l}0.560 \\
0.649\end{array}$ & $\begin{array}{l}0.634 \\
0.744\end{array}$ \\
\hline$M 3 b=$ & $\left(\begin{array}{lll}0.3 & 0.6 & 0.1 \\
0.1 & 0.3 & 0.6 \\
0.6 & 0.1 & 0.3\end{array}\right)$ & 0.56 & 0.3 & $\begin{array}{l}0.05 \\
0.10\end{array}$ & $\begin{array}{l}0.462 \\
0.623\end{array}$ & $\begin{array}{l}0.475 \\
0.609\end{array}$ & - \\
\hline$M 3 c=$ & $\left(\begin{array}{lll}0.1 & 0.6 & 0.3 \\
0.3 & 0.1 & 0.6 \\
0.6 & 0.3 & 0.1\end{array}\right)$ & 0.56 & 0.1 & $\begin{array}{l}0.05 \\
0.10\end{array}$ & $\begin{array}{l}0.837 \\
0.918\end{array}$ & $\begin{array}{l}0.860 \\
0.918\end{array}$ & - \\
\hline
\end{tabular}

can be decomposed into three independent NHPPs. Since a model assumption is the independence of the three marginal NHPPs, the proposed test is applied in its validation analysis. 
4.1 Common Poisson shock process

A common Poisson shock process (CPSP), denoted by $N$, is a multivariate point process which assumes that there is an underlying PP of shocks $N_{0}$, that can yield $d$ different types of events. The counting process of the $j^{\text {th }}$ type of events, for $j=$ $1, \ldots, d$, is the Poisson component process $N_{j}$ (Lindskog and McNeil 2003).

For the bivariate case, $d=2$, events can be divided into 3 types, $(1,0),(0,1),(1,1)$, depending on the components where the occurrence has been observed. Given two component processes $N_{1}$ and $N_{2}$, see Figure 3, the bivariate process can be decomposed into three independent processes: $N_{(1)}$ which includes the events occurring only in the $N_{1}$ process, $N_{(2)}$ which includes the events occurring only in $N_{2}$, and $N_{(12)}$ which contains the events occurring simultaneously in $N_{1}$ and $N_{2} . N_{(1)}, N_{(2)}$ and $N_{(12)}$ are the marginal processes with intensities $\lambda_{(1)}, \lambda_{(2)}$ and $\lambda_{(12)}$. The distribution of a CPSP is completely specified by its marginal processes. This decomposition into independent PPs allows the model to be readily applied for data modeling and easily generalizable to the $\mathrm{NH}$ case, by permitting the marginal intensities to be a function of time-varying covariates. These covariates also model the dependence induced by the systematic part of the intensities and, consequently, the independence between the processes becomes conditional independence, given the covariates.

\subsection{Data}

The daily maximum and minimum temperature series of Zaragoza (Spain) from May to September (MJJAS) for the period [1951, 2004], $T x$ and $T n$ herein, are used for 
20
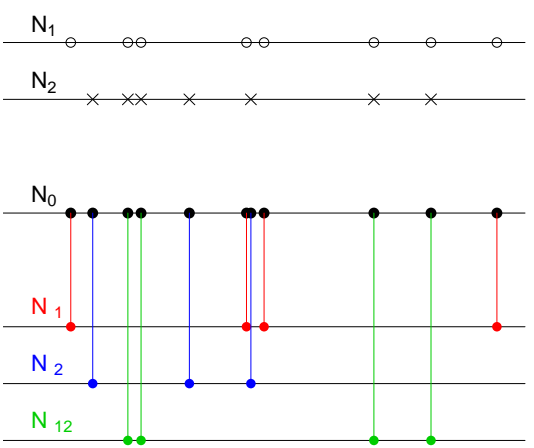

Fig. 3 Decomposition of the CPSP into three independent marginal Poisson process

illustration. Data are provided by AEMET, the Spanish meteorological office. A thorough exploratory analysis shows a time evolution not seasonally homogeneous, and a time-varying dependence between the occurrence of both type of extremes. A measure summarizing the extremal dependence between two variables is the coefficient $\chi=\lim _{u \rightarrow 1} \chi(u)$, with $\chi(u)=P(U>u \mid V>u)$ and $(U, V)$ the transformed uniform marginals (Coles et al. 1999). For $T x$ and $T n, \hat{\chi}(0.95)=0.43$ and $\hat{\chi}=0.2$ while, under independence, they should be 0.05 and 0 , respectively. Under these conditions, two different but dependent models for describing the occurrence of the $T x$ and $T n$ extremes are required, and the suggested NHCPSP provides an adequate flexible framework.

\subsection{Extreme event definition}

An extreme heat event (EHE) only in $T x$ is a run of consecutive days where $T x$ exceeds its extreme threshold $U_{x}$ but $T n$ does not exceed its extreme threshold $U_{n}$. An EHE only in $T n$ is defined analogously. A simultaneous EHE is a run where $T x$ and $T n$ exceed $U_{x}$ and $U_{n}$, respectively. In Zaragoza, these thresholds are $U_{x}=37.0^{\circ} \mathrm{C}$ 
and $U_{n}=21.2^{\circ} \mathrm{C}$, the $95 \%$ percentiles in the reference period (months from June to August in 1971-2000). Tools for selecting adequate threshold can be found in Coles (2001) and a specific justification for the use of the $95 \%$ percentile in temperature series in Abaurrea et al. (2007).

In the EHE only in $T x$ or $T n$, the occurrence point is located at the maximum temperature time and, in the simultaneous events, at the time where the sum of both temperatures is maximum. In the Zaragoza temperature series, there are 120 EHEs only in $T x, 92$ only in $T n$ and 58 simultaneous EHEs.

4.4 Estimation of the model

The estimation of a NHCPSP reduces to the estimation of the three marginal NHPPs, whose intensities are modeled as parametric functions of time dependent covariates. An important application of this model is to obtain projections of the occurrence of EHEs in climate change scenarios. For that reason, we need covariates whose future $21^{\text {st }}$ century values should be available to feed the model. Since General Circulation Model projections for temperature are reliable, but only at an aggregated time scale (Abaurrea et al. 2007) the following covariates were considered:

- Temperature terms. Two possible influential factors on EHEs are the current temperature level (short term temperature) and the decadal temperature trend (long term temperature). The short term signal is defined as the moving average in 15 or 31 day intervals around day $t$, and denoted $T x_{m 15}, T n_{m 15}, T x_{m 31}$ and $T n_{m 31}$. The long term covariates, $T T x$ and $T T n$, are the lowess with a $30 \%$ 
22

window of the daily temperature series. Polynomial terms are also considered to model possible non linear effects.

- Seasonal terms. The part of annual harmonics corresponding to the five summer months, defined by the pairs of covariates $\cos (2 k \pi t)$ and $\sin (2 k \pi t)$ with $t=121 / 365, \ldots, 273 / 365$ (the time index from $1^{s t}$ of May to $30^{t h}$ of September) and $k=1,2, \ldots$

- Interaction terms. Harmonic and temperature interaction terms allow us to model changing effects of temperature over the summer.

Parameters are estimated by conditional (on the covariates) maximum likelihood; the conditional loglikelihood of a NHPP can be found in Coles (2001). A logarithmic link is used to guarantee positive intensities. To keep the notation simple, the conditional intensity $\lambda(t \mid \mathbf{X}(\mathbf{t}))=\exp \left(\mathbf{x}^{\prime}(\mathbf{t}) \beta\right)$ is denoted by $\lambda(t)$, being $\mathbf{X}(\mathbf{t})=$ $(\mathbf{x}(\mathbf{1}), \ldots, \mathbf{x}(\mathbf{t}))^{\prime}$ the covariate matrix and $\mathbf{x}(\mathbf{t})$ the vector of covariates at time $t$. It is worth to recall that efficient estimation based only on the conditional model (ignoring the information of the marginal process), as we do, can only be carried out if weak exogeneity holds (Ericsson and Irons 1994, chap. 1). The weak exogeneity of the covariates in our model has been checked using the definition given by (Engle et al. 1983, p. 282).

Covariate selection is based on likelihood ratio tests with a $\alpha=0.05$ significance level. The final intensities fitted to the three marginal processes are,

$$
\begin{aligned}
\log \left(\lambda_{(1)}(t)\right)= & -68.9-47.6 \cos (2 \pi t)-22.6 \sin (2 \pi t)+0.08 T x_{m 15}+0.21 T n_{m 15}+ \\
& 0.25 T n_{m 15} \cos (2 \pi t)+0.12 T n_{m 15} \sin (2 \pi t)+0.01 T T x \\
\log \left(\lambda_{(2)}(t)\right)= & -13.3-0.35 \cos (2 \pi t)+0.26 \sin (2 \pi t)+0.11 T n_{m 15}-0.02 T x_{m 31}
\end{aligned}
$$




$$
\begin{aligned}
\log \left(\lambda_{(12)}(t)\right)= & -32.1-6.6 \cos (2 \pi t)-3.1 \sin (2 \pi t)+0.01 T n_{m 15}+0.04 T x_{m 15}+ \\
& 0.07 T T n
\end{aligned}
$$

4.5 Validation analysis

The assumptions to be checked are the NHPP behavior of the three marginal processes and their mutual independence.

Checking the Poisson character. The usual approach to check a univariate $\operatorname{NHPP}(\lambda(t))$ is to test whether the process after the time transformation $t^{*}=\int_{0}^{t} \lambda(u) d u$ is a unit rate HPP. Then, the validation analysis is standard and it is based on the study of the uncorrelation and the exponentiality of the inter-event distances $d_{i}^{*}=t_{i}^{*}-t_{i-1}^{*}$, or the uniform character of $\exp \left(-d_{i}^{*}\right)$. A more detailed example of the application of these validation techniques can be found in Abaurrea et al. (2007). The point process raw residuals (Baddeley et al. 2005) are also checked.

The validation results, briefly summarized here, do not show any evidence against the fitted model: the p-values of the uniform KS test are 0.56 for $N_{(1)}, 0.16$ for $N_{(2)}$ and 0.55 for $N_{(12)}$, and all the Kendall correlation p-values are greater than 0.7 . The empirical and the fitted rates $\int_{t_{1}}^{t_{2}} \hat{\lambda}(u) d u /\left(t_{2}-t_{1}\right)$, both calculated in moving 5-month intervals, are plotted in Figure 4 . For $N_{(1)}$, its lowess with a $20 \%$ window is also shown (top right plot). The observed evolution is satisfactorily reproduced by the fitted intensity, and a clear increase of the occurrence rate from the late 90 's is observed. 
Concerning the other processes, an increase of the occurrence rate, higher in $N_{(2)}$, is observed from the 80 's, and it becomes steeper from the 90 's onwards.
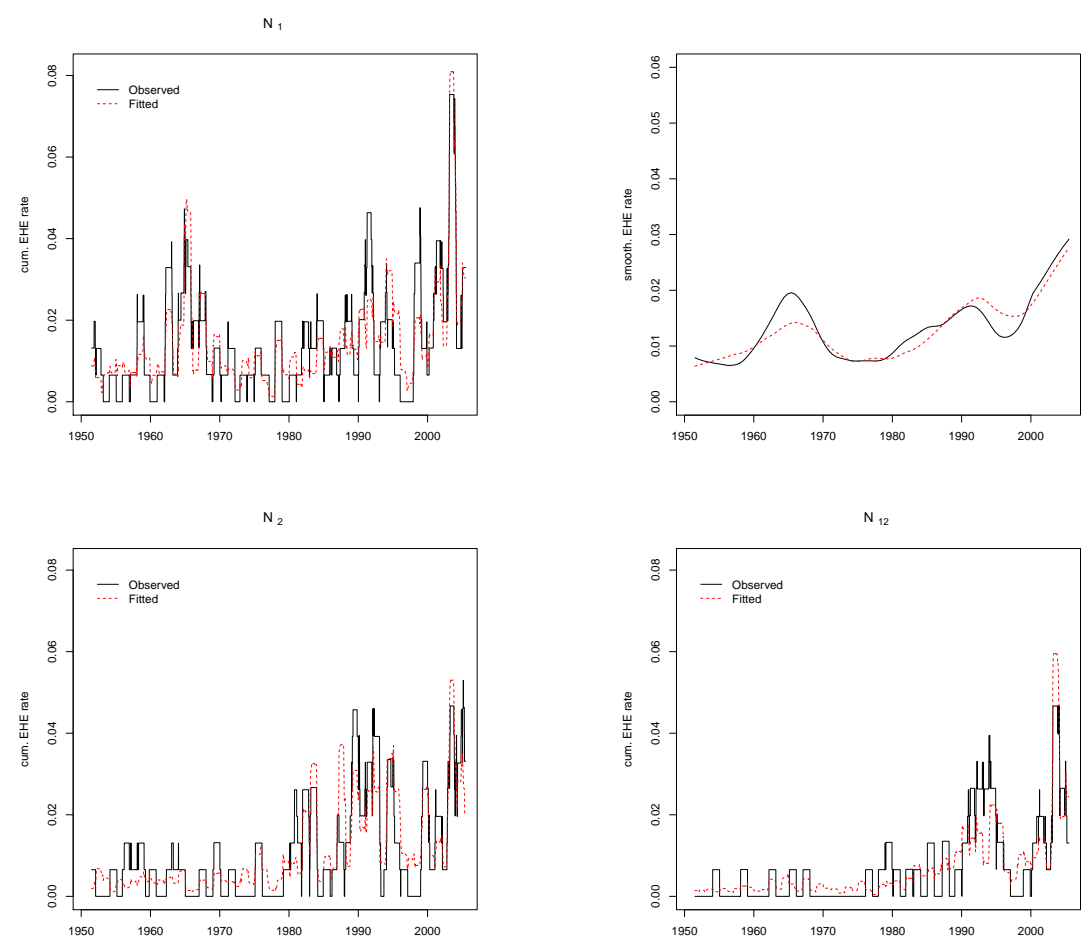

Fig. 4 Empirical and fitted intensities of the marginal processes of the NHCPSP for the extreme heat events in Zaragoza. The top right plot is the $20 \%$ lowess of the signals of $N_{(1)}$

Checking the independence of the marginal processes. To check the independence between $N_{(1)}, N_{(2)}$ and $N_{(12)}$, the test in Section 2.1 is applied with $n_{S}=1000$ simulations. Since the real intensities of the PP are unknown, a parametric bootstrap p-value is calculated using the MLE $\hat{\lambda}_{y}(t)$ and $\hat{\lambda}_{z}(t)$, estimated under the null hypothesis. The marginal PP have been satisfactorily validated, so that it can be assumed that the in- 
tensities are well estimated. The p-value of the independence test is 0.423 when close points are defined as points with overlapping intervals, and 0.569 when the previous and the following points are included in the definition. In both cases, the null hypothesis cannot be rejected at any usual significance level, and the three processes can be considered independent.

\section{Conclusions}

A test to check the independence between NH point processes is developed. When the parameters describing the point processes are known, it is a Monte Carlo test but, in most real problems, the parameters are unknown and it is a parametric bootstrap test. The test is developed for the three-process case, but it can be generalized to any number of processes. It is noteworthy that it can be used to check the independence of any point process which can be simulated, since the type of process (Poisson for example) is only used to generate independent trajectories.

The use of a computational algorithm which reuses the generated processes in two steps of the test (the calculation of the distance percentiles and the distribution of the test statistic) allows us to keep low the computation time.

A simulation study shows that the size of the test reaches its nominal level and that its power is satisfactory and improves with the sample size. Two approaches for generating dependent NH processes are considered: NHPP based on two queues in tandem and NH point processes resulting from marking a PP using a Markov chain. Different measures for quantifying the dependence between the $\mathrm{NH}$ processes are suggested. 
26

This test is a necessary tool in the validation analysis of a common Poisson shock process for checking the independence of the marginal Poisson processes. It could also be used in different applications to check the independence between point processes characterizing the time evolution of different variables. This type of problems appear in biomedical sciences, economy and specially in environmental sciences, for example to represent the occurrence of earthquakes in several regions.

Concerning the temperature application, it is shown that the CPSP is a valuable model to characterize the occurrence of extreme events in two dependent variables. It is successfully applied to model the occurrence of EHEs in daily maximum and minimum temperatures.

Acknowledgements This work was partially supported by Ministerio de Educación y Ciencia (Spanish Department of Science) and Ministerio de Medio Ambiente (Spanish Department of Environment) through the projects CGL2009-09646 and ESTCENA 2009/0017.

\section{References}

Abaurrea, J., Asín, J., Cebrián, A. C., and Centelles, A. (2007), " Modeling and forecasting extreme heat events in the central Ebro valley, a continental-Mediterranean area," Global Planet. Change, 57(1-2), 43-58.

Allard, D., Brix, A., and Chadoeuf, J. (2001), "Testing local independence between two point processes," Biometrics, 57, 508-17.

Andrews, D. W. K. (1997), ”A conditional Kolmogorov test,” Econometrica, 65(5), 1097-128.

Beran, R. (1988), "Prepivoting Test Statistics: A Bootstrap View of Asymptotic Refinements," J. Am. Stat. Assoc., 83(403), 687-97.

Baddeley, A. J., Møller, J., and Waagepetersen, R. (2000), ”Non-parametric and semi-parametric estimation of interaction in inhomogeneous point patterns," Stat. Neer., 54, 329-50. 
Baddeley, A., Turner, R., Møller, J., and Hazelton, J. (2005), ’Residual analysis for spatial point processes,” J. R. Statist. Soc. B, 67(5), 617-66.

Coles, S. (2001), An introduction to statistical modeling of extreme values, Springer.

Coles, S., Heffernan, J., and Tawn, J. (1999), ’Dependence measures for extreme value analysis," Extremes, 2(4), 339-65.

Comas, C., Mateu, J., and Särkkä, A. (2010), ”A third-order point process characteristic for multi-type point processes," Stat. Neer., 64, 19-44.

Cuzick, J., and Edwards, R. (1990), "Spatial clustering for inhomogeneous populations," J. Roy. Statist. Soc., B, 52, 73-104.

Daley, D., and Vere-Jones, D. (2003)', An introduction to the theory of point processes. Vol I: Elementary theory and methods, Springer.

Davidson, R., and MacKinnon, J. G. (1999), "The size distortion of bootstrap tests, " Economet. Theor., $15,361-76$

Davidson, R., and MacKinnon, J. G. (2006), ’Bootstraps methods in Econometrics," in Palgrave Handbook of Econometrics: Volume 1 Theoretical Econometrics, K. Patterson and T. C. Mills (eds.), Basingstoke, Palgrave Macmillan, pp 812-38.

Davidson, R., and MacKinnon, J. G. (2006), 'The power of bootstrap and asymptotic tests," J. Econometrics, $133,421-41$.

Diggle, P. J. (1983), Statistical analysis of spatial point patterns, Academic Press.

Doss, H. (1989), ’On estimating the dependence between two point processes,” Ann. Stat., 17(2), 749-63.

Dufour, J. M. (2006), ’Monte Carlo test with nuisance parameters: a general approach to finite-sample inference and nonstandard asymptotics," J. Econometrics, 133, 443-77.

Eckel, S., Fleischer, F., Grabarnik, P., and Schmidt, V. (2007), ”An investigation of the spatial correlations for relative purchasing power in Baden-Württemberg," Adv. Stat. Anal., 92, 135-52.

Engle, R. F., Hendry, D. F., and Richard, J. F. (1983), ’Exogeneity,” Econometrica, 51(2), 277-304.

Ericsson, N. R., and Irons, J. S. (1994), Testing exogeneity, Oxford University Press.

Grintzevitch, S. (2006), Heat waves: their climatic and biometeorological nature in two north american regions, $\mathrm{Ph}$. D. dissertation, University of Reading, School of Mathematics, Meteorology \& Physics, http://www.reading.ac.uk/web/FILES/maths/sarah_grintzevitch.pdf 
Guan, Y. and Afshartous, D. (2007). Test for independence between marks and points of marked Environ. Ecol. Stat., 14, 101-11.

Horowitz, J. L. (2001), "The Bootstrap," in Handbook of Econometrics, J. J. Heckman and E. E. Leamer (eds.), edition 1, vol. 5, 3159-228, Elsevier.

Isham, V. (1980), "Dependent thinning of point processes," J. Appl. Probab., 17(4), 987-95.

Keilson, J., and Servi, L. D. (1994), "Networks of non-homogeneous $M / G / \infty$," J. Appl. Probab., 31, Studies in Applied Probability, 157-68.

Kleinrock, L. (1975), Queueing systems. Vol. I: Theory, Wiley.

Lieshout, M. N. M. van, and Baddeley, A. J. (1999), ”Indices of dependence between types in multivariate point patterns,” Scand. J. Stat., 26(4), 511-32.

Lindskog, P., and McNeil, A. (2003), "Common Poisson shock models: applications to insurance and risk modelling," ASTIN Bulletin, 33, 209-38.

Marcon, E., and Puech, F. (2009), "Generalizing Ripley's K function to inhomogeneous populations," Working papers Halshs, 00372631-v1, HAL.

Palecki, M. A., Changnon, S. A., and Kunkel, K. E. (2001), ”The nature and impacts of the July 1999 heat wave in the midwestern United States: learning from the lessons of 1995," B. Am. Meteorol.l Soc., 82, 1353-67.

Plummer, N., Salinger, M., Nicholls, N., Suppiah, R., Hennessy, K., Leighton, R., Trewin, B., Page, C., and Lough, J. (1999), "Changes in climate extremes over the Australian region and New Zealand during the Twentieth Century," Clim. Change, 42, 183-202.

Schlather, M., Ribeiro, P. J., and Diggle, P. J. (2004), ’Detecting dependence between marks and locations of marked point processes," J. R. Statist. Soc. B, 66(1), 79-93. 


\section{Bio-note}

Dr. Ana C. Cebrián is senior lecturer at the Department of Statistics of the University of Zaragoza. Her background is on Mathematics and she completed her doctoral dissertation on extreme value theory with application on drought process modelling. Since 1993, she has been teaching at the Faculty of Sciences, first as assistant and associate professor and then as senior lecturer (2003). After obtaining the Prix FSR 2000 from the Catholic University of Leuven, she occupied a 1-year post-doctoral position at the Institute of Statistics of that university. At present, she is working with the Stochastic Processes research group and more specifically on the development of statistic and probability models for climate data.

Research

The general topic of my research is statistical modelling applied to climate data. More specifically, it focuses on extreme value analysis; that is the development of statistical and probabilistic models to handle extreme data. I apply these models to the analysis of drought, periods with extremely low rainfall, or heat waves, periods with extremely high temperatures. The objective is to design models capable of describing not only what has happened in the past but also to use information to make long term forecasts of the characteristics of the extreme events in the future. Currently, I am working with bivariate models based on copulas. 